Greece

\title{
MANIPULATION OF A SHOCK-WAVE/BOUNDARY-LAYER INTERACTION IN THE TRANSONIC REGIME AROUND A SUPERCRITICAL MORPHING WING
}

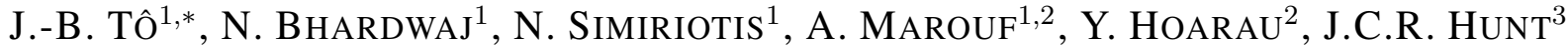 \\ AND M. BRAZA ${ }^{1, \dagger}$ \\ ${ }^{1}$ Institut de Mécanique des Fluides de Toulouse, Allée du Professeur Camille Soula, Toulouse, France \\ ${ }^{2}$ Institut de Mécanique des Fluides et des Solides de Strasbourg, Strasbourg, France \\ ${ }^{3}$ University College London, London, UK \\ *jean-baptiste.to@imft.fr \\ †marianna.braza@imft.fr
}

\begin{abstract}
.
Wing morphing as a means to continuously modify wing geometry is employed in a numerical simulation in order to act upon aerodynamic performance in cruise flight. In this flight regime, the interaction of a shock wave and boundary layer instabilities can lead to transonic buffet, the coordinated motion of both the shock and the detached boundary layer. This flow instability induces unsteady forces on the wing which may induce dip-flutter or cause stall. Understanding where it comes from and how to act on it can be crucial for extending flight envelope or reach better fuel efficiency in the transonic regime. It has been observed that a compliant trailing edge can alter the amplitude and frequency of the buffet instability and improve aerodynamic performance by modifying the flow topology of the wake and detached boundary layer.
\end{abstract}

Key words: instability, buffet, OES, supercritical wings, compressible flow.

\section{Nomenclature}

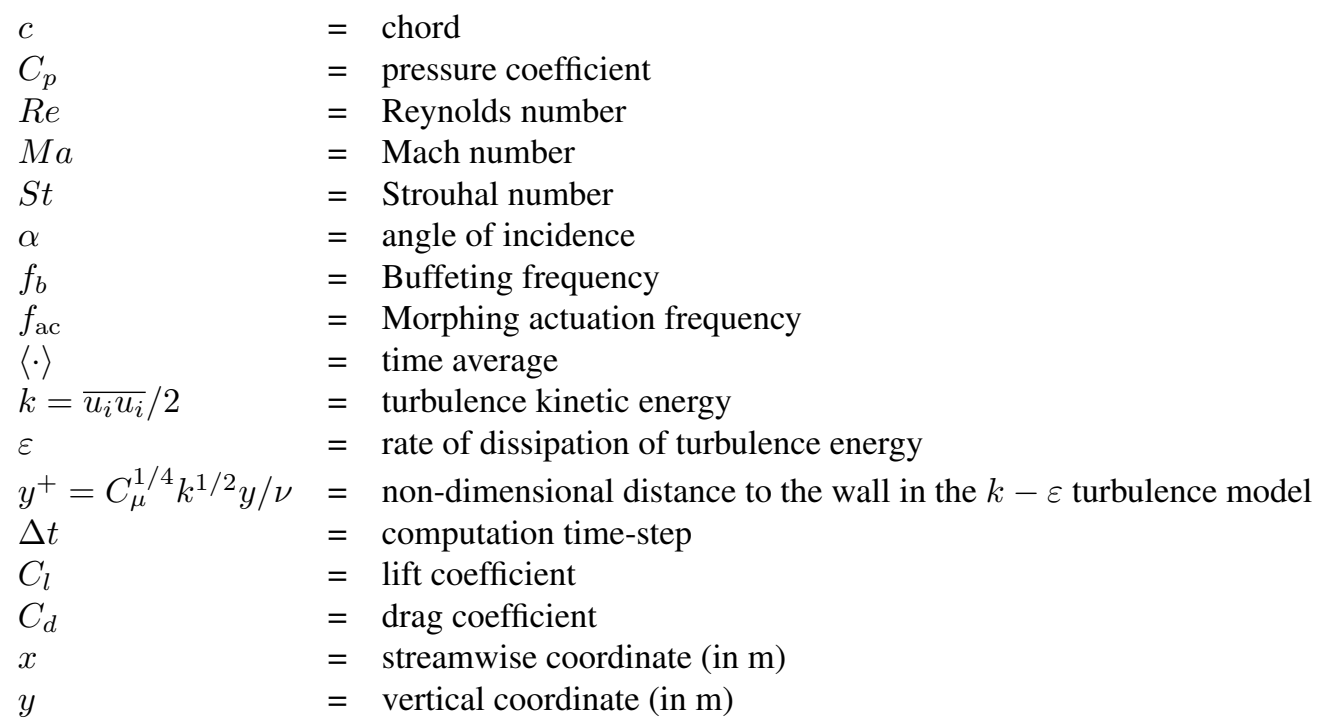




\section{Introduction}

The main purpose of this paper is to study the influence of wing morphing on the aerodynamic behaviour of a supercritical wing at high Reynolds numbers in the transonic regime - that is for Mach numbers between 0.7 and 1.2. In these flight regimes and for certain angles of attack, the acceleration of air over a wing can be such that the airflow reaches supersonic speeds over the wing even for Mach numbers lower than 1 . This acceleration results in flow compression, marked by the appearance of a normal shock or a lambda shock. Due to the reverse pressure gradient caused by flow compression at the shock foot, the boundary layer is made to thicken and may separate significantly. As the turbulent boundary layer detaches, the rear flow geometry is considerably modified, with the thickening of the shear layer and wake region negatively contributing to aerodynamic performance due to an increase of pressure drag and viscous drag.

The interaction of the shock and the boundary layer that detaches aft the shock may result in an oscillating motion of the entire interaction zone, causing the shock to move back and forth and the boundary layer to alternatingly thicken up and thin down. This flow instability is called transonic buffet. Over the past forty years, numerous analyses have been made to understand and predict the occurrence of buffet, with pioneering studies by H.L. Seegmiller et al. (1978), McDevitt et al. (1976), Levy Jr. et al. (1978) or even Marvin et al. (1980), investigating the behaviour of this instability using Schlieren imaging and early computational results.

In 2012, a study by Grossi et al. (2012) demonstrated how a splitter-plate placed at the aft end of a wing stifles the Von Kármán mode, and weakens the buffet instability. A feedback mechanism from the rear part of the flow — including shear layer instabilities in the detached boundary layer and the Von Kármán instability in the wake — towards the shock has thus been highlighted.

The previous observations indicate that it should be possible to reduce the intensity of buffet or manipulate the unsteady aerodynamic loads thanks to morphing, i.e. the continuous transformation of the wing's shape. The present study investigates the effects of a trailing edge monochromatic vibration on the frequency of buffet. Hybrid morphing, as a combination of a single trailing edge deflection and high-frequency vibrations, has also been studied. Finally, it is shown how aerodynamic performance can be impacted by the frequency of actuation and how drag reduction can be achieved through dynamic morphing.

\section{Numerical setup}

The numerical domain used in this study is a two-dimensional 190,000-cell structured, C-H topology multiblock mesh (cf. fig. 2) around an A320 airfoil of chord $c=0.15$ $\mathrm{m}$. This geometrical configuration comes from a collaboration between several research teams among the European project SMS - Smart Morphing and Sensing for aeronautical configurations. As such, the flow parameters and size of the wing in the numerical study have to be in accordance with those of the transonic windtunnel in the IMP-PAN Academy of Science of Gdansk, Poland. A Reynolds number of $R e=2.07 \times 10^{6}$ and a Mach 
number of $M a=0.78$ are chosen to reproduce cruise flight conditions.

Two angles of attack were investigated in this paper. While most quantitative results have been generated for $\alpha=1.8^{\circ}$ which is the standard configuration for high altitude cruise flight, an incidence angle of $\alpha=5^{\circ}$ has also been studied due to the stronger SWBLI effects that appear with that angle, in order to better understand the large scale unsteadiness due to buffet.

A URANS approach was chosen as an ideal trade-off between computational speed and accuracy, with a $k-\varepsilon$ Chien model used along the Organised Eddy Simulation (OES) statistical approach as in Jin and Braza (1994), Braza et al. (2006), Szubert et al. (2015) in order to correctly represent and predict the complex dynamics of coherent structures and chaotic turbulence.

(a)
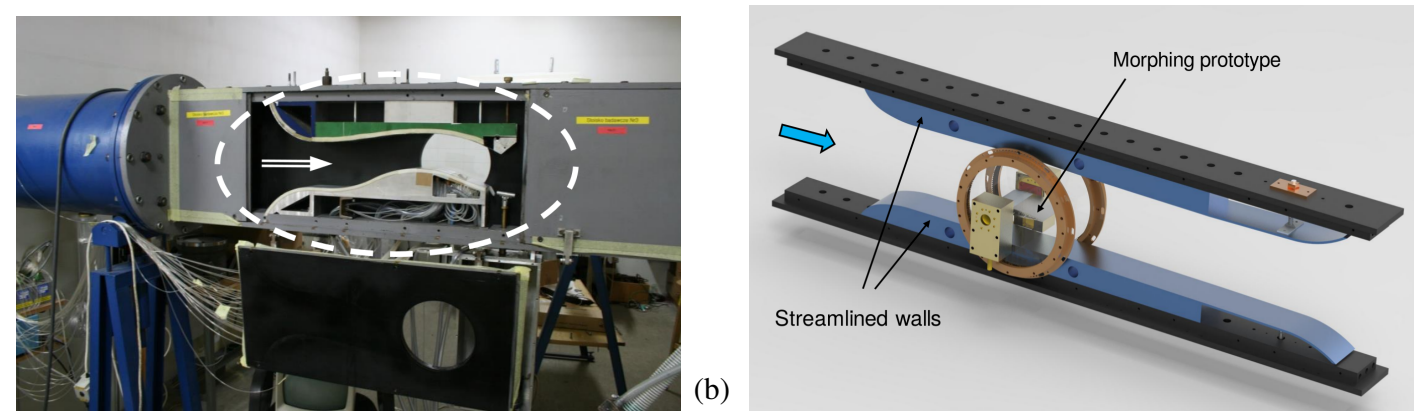

Figure 1: a) Transonic windtunnel test section in IMP-PAN b) The morphing wing test bed with streamlined walls above and below the wing.

Thanks to the collaboration between our research group and other partners within the NSMB European Consortium, transonic computations were run using the Navier-Stokes Multi Block (NSMB) code (see Hoarau et al. (2016)). Numerical results are found by solving the compressible Navier-Stokes equations using a finite-volume formulation. A third-order Roe upwind scheme has been employed for the discretization of spatial fluxes while time integration was performed through a dual-time stepping, second-order backward difference scheme as per past studies by Szubert et al. (2015), Grossi et al. (2012) and Tô et al. (2019). For time-accuracy, a time-step of $\Delta t=5 \times 10^{-6}$ has been chosen.

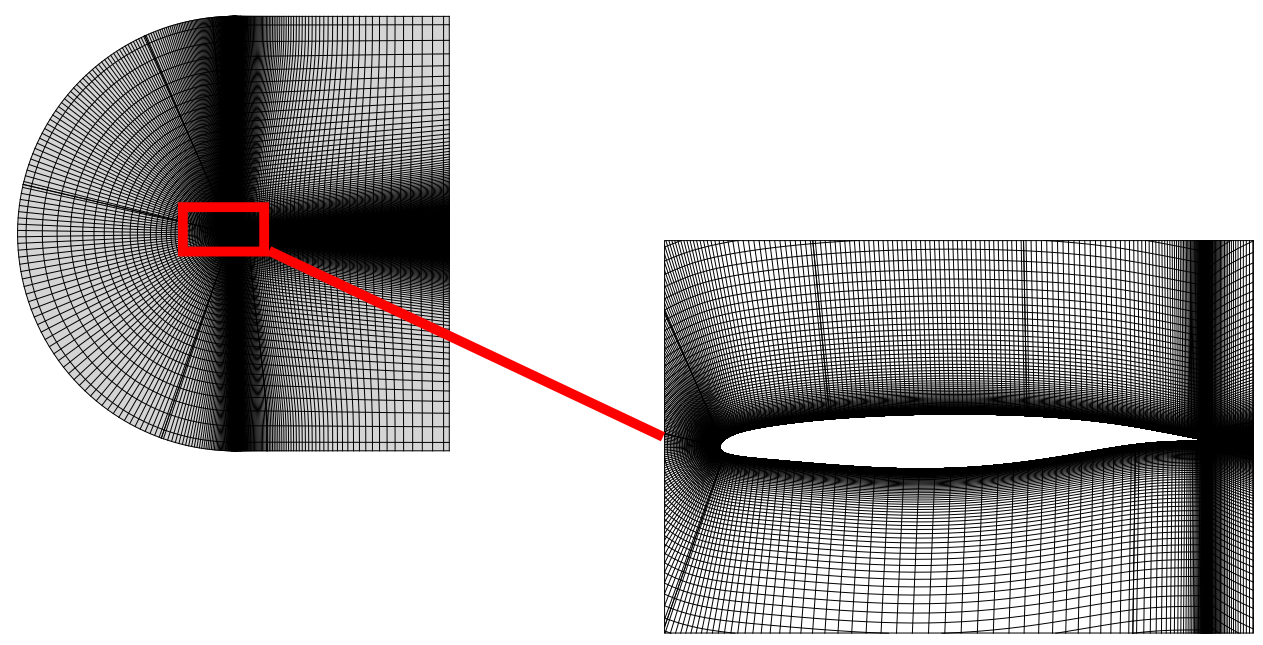




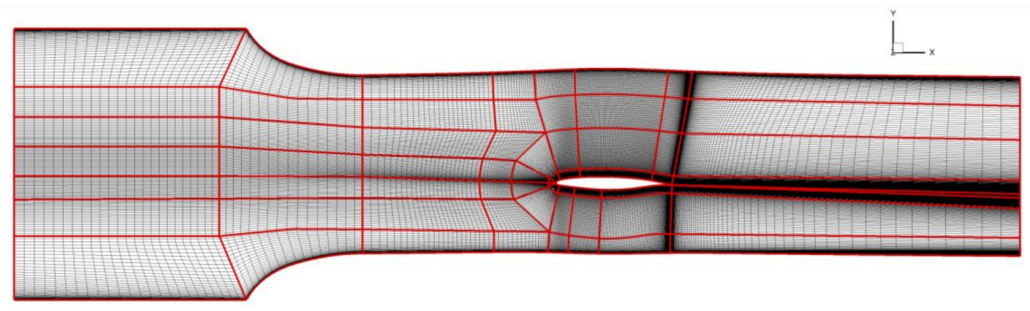

Figure 2: Above: 2D structured multiblock mesh; Below: "Numerical windtunnel" 3D structured multiblock mesh

\section{Results}

\section{Effect of morphing on the force coefficients}

When integrated over the surface of the wing, the unsteady loads due to the SWBLI result in a quasi-periodic signal. This is mainly due to the motion of the shock that causes the compression zone to oscillate back and forth, explaining the relatively gentle slope at $x / c \approx 0.7$ in the time-averaged $-C_{p}$ profile of fig 3 . Indeed, if the shock was still, the pressure distribution would be steeper in the shock area. The detached boundary layer also increases pressure aft the shock as highlighted by the green ellipse in the same figure. This is due to the strong reverse pressure gradients that accompany shock-induced flow separation.

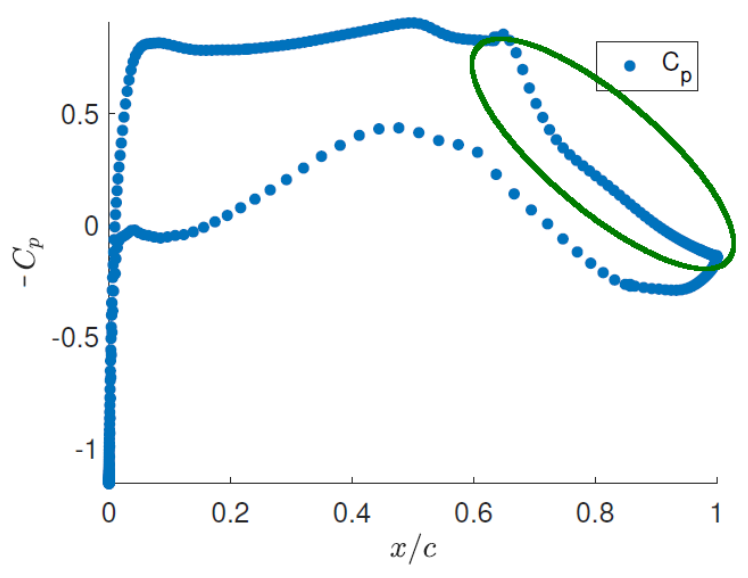

Figure 3: Time-averaged pressure coefficient plot for the static configuration.

It is seen, through the red curve of figure 5 that a slight upward deflection of the trailing edge by $2^{\circ}$ can annihilate flow unsteadiness due to buffet, thus proving that buffet can be suppressed, although at a loss of lift. Indeed, an upward motion of the trailing edge lowers wing curvature along the suction side, thus reducing the intensity of the reverse flow and as a consequence diminishes both the thickness and extent of the detached boundary layer. Figure 4 shows an instance of strong flow separation and destabilization due to SWBLI.

Additionally, the lift coefficient time series for a combined deflection and vibration at $f_{\mathrm{ac}}=90 \mathrm{~Hz}$ (blue curve of fig. 5) shows that the average value of the lift coefficient is 


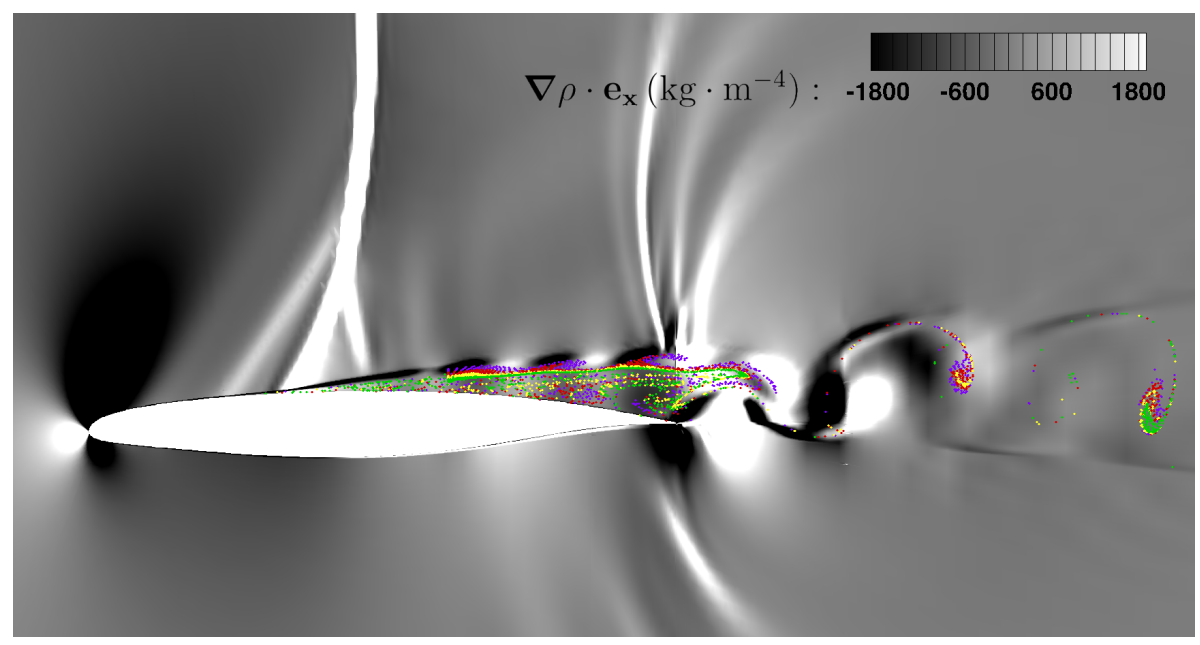

Figure 4: Density gradient iso-contours and streaklines around an airfoil at $M a=0.78$, for an angle of incidence $\alpha=5^{\circ}$. The presence of the Von Kármán instability and the destabilization of the shear layer aft the shock are symptomatic of a strong SWBLI. The same behaviour can be observed, to a lesser extent, for $\alpha=1.8^{\circ}$.

increased compared to that of a simple deflection of the trailing edge. This implies that adding a vibration to the trailing edge allows to modify the average values of both lift and drag by changing the flow dynamics of the turbulent viscous zones, namely the shear layer and turbulent wake.

Notably, we observe that although average lift is decreased in the case of an upward deflection, average drag is decreased by a more substantial amount. Overall, time-averaged lift to drag ratio is increased by $10.4 \%$. It has been observed that lift to drag ratio increases in all morphing types, for both angles of attack $\alpha=1.8^{\circ}$ and $\alpha=5^{\circ}$, even though this increase is more important for small incidences. In fact, due to the strong separation that happens for important angles of attack, small modifications of the trailing edge geometry or small vibration have a lesser impact on aerodynamic performance. It has been shown by Jodin et al.? that hybrid morphing with high-amplitude wing distortions is particularly effective for low-Mach regimes at large angles of incidence.

These results are listed in the table below:

\begin{tabular}{|c|c|c|c|c|}
\hline Incidence & \multicolumn{2}{|c|}{ Angle of attack 1.8 } & \multicolumn{2}{c|}{ Angle of attack 5 } \\
\hline Type of actuation & $\mathrm{D}$ & $\mathrm{D}+\mathrm{F}$ & $\mathrm{D}$ & $\mathrm{D}+\mathrm{F}$ \\
\hline$\frac{\left\langle C_{l} / C_{d}\right\rangle-\left\langle C_{l} / C_{d}\right\rangle_{\text {static }}}{\left\langle C_{l} / C_{d}\right\rangle_{\text {static }}} \times 100$ & $+10.4 \%$ & $+4.3 \%$ & $+2.5 \%$ & $+0.4 \%$ \\
\hline
\end{tabular}

Table 1: Relative mean lift over drag ratio gain compared to the static case. Note that D means " $2^{\circ}$ deflection" and "D+F" is the superposition of an immobile upward deflection and a $90 \mathrm{~Hz}$ flapping motion. 


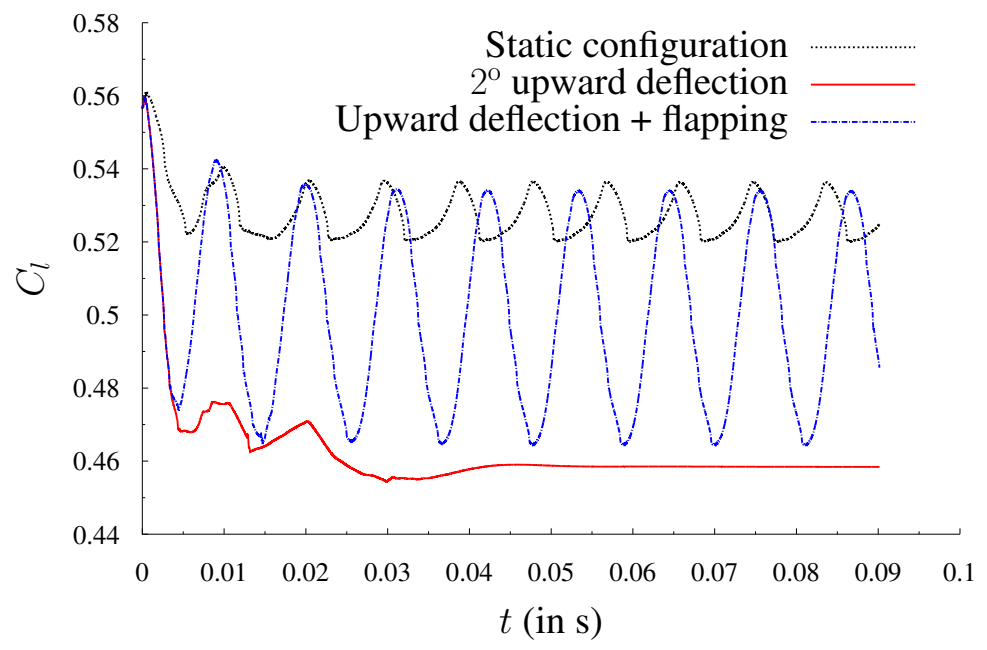

Figure 5: Lift coefficients for $\alpha=1.8^{\circ}$

\section{Frequency synchronization}

For the entire array of frequencies $\mathcal{F}:=\{100 ; 150 ; 200 ; 250 ; 300 ; 350 ; 400 ; 450 ; 500 ; 700$; $720 ; 750 ; 800 ; 1000 ; 1500\} \mathrm{Hz}$, the vibrating trailing edge appears to synchronize the SWBLI frequency as is shown in fig. 5 and some Power Spectral Density plots below. The first peak, corresponding to the buffet mode, appears to be shifted in the frequency domain and perfectly coincides with the frequency of trailing edge actuation:

As can be observed in figure 6, the leftmost mode represented by a wide bump corresponds to the natural buffet frequency $f_{b}=111 \mathrm{~Hz}$ (for a Strouhal number $S t=$ $6.65 \times 10^{-2}$ ) in the case of an immobile wing (black curve). When the trailing edge of the wing vibrates at a certain frequency $f_{\text {ac }}$, the up-and-down motion of the geometry walls exerts a periodic pressure disturbance in the boundary layer which forces it to move along with the trailing edge. The separation zone is thus made to travel back and forth which causes compression waves near the separation zone - and consequently the shock — to develop an oscillatory motion that is synchronized with the vibrating trailing edge.

\section{Time-averaged force coefficients at various actuation frequencies}

The mean values of the aerodynamic force coefficients as well as their RMS are found for frequencies $f \in \mathcal{F}$. The actuation considered is a monochromatic flapping actuation around the static trailing edge with an angle of flapping $\alpha_{F}=1^{\circ}$.

The lift coefficient is seen to increase compared to the static case for $f_{\mathrm{ac}}=400 \mathrm{~Hz}$, $f_{\mathrm{ac}}=450 \mathrm{~Hz}, f_{\mathrm{ac}}=500 \mathrm{~Hz}$ and $f_{\mathrm{ac}}=720 \mathrm{~Hz}$ while drag decreases compared to the static case for all frequencies but $f_{\mathrm{ac}}=400 \mathrm{~Hz}, f_{\mathrm{ac}}=450 \mathrm{~Hz}$ and $f_{\mathrm{ac}}=500 \mathrm{~Hz}$. This shows that the only actuation frequency among those that were studied that both increases lift and decreases drag is $f_{\mathrm{ac}}=720$.

It is noticed that mean lift and drag follow the same trends. This suggests that the underlying mechanisms explaining lift and drag generation through vibrational trailing edge morphing is the same. 

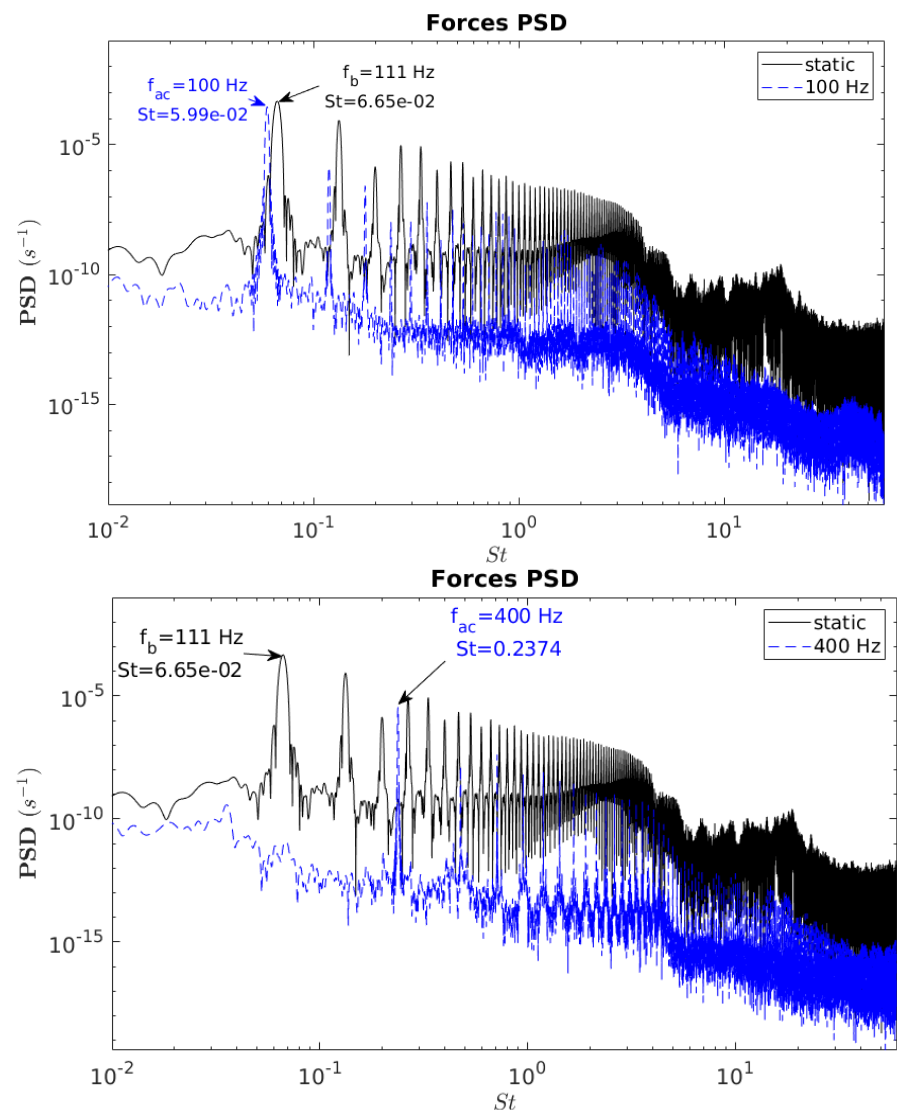

Figure 6: Power Spectral Density plots of the lift coefficient for $f_{\mathrm{ac}}=100 \mathrm{~Hz}$ (above) and for $f_{\text {ac }}=400$ (below). The black curves represent the PSD estimation via a Welch method applied to the lift coefficient of the unmorphed wing. The blue curves stand for morphing cases.

Some frequencies stand out in terms of performance improvement. In particular, it is observed that an actuation frequency of $f_{\mathrm{ac}}=720$ decreases drag by $1.2 \%$ and increases lift by $0.3 \%$, cf. figure 8 . Although lift is decreased by $1.1 \%$ and $3.8 \%$ for $f_{\text {ac }}=300 \mathrm{~Hz}$ and $f_{\mathrm{ac}}=350 \mathrm{~Hz}$ respectively, drag is reduced by $3.5 \%$ for $f_{\mathrm{ac}}=300 \mathrm{~Hz}$ and by $8.9 \%$ for $f_{\mathrm{ac}}=350 \mathrm{~Hz}$. This signifies that lift to drag ratio increases by up to $5.5 \%$ in the case of an actuation frequency of $350 \mathrm{~Hz}$.

\section{Comparison of mean velocity profiles in the wake}

The wake velocity profile at $x / c=0.19$ for frequencies 300,350 and $400 \mathrm{~Hz}$ has been compared with the static case. The wake width is calculated from figure 9 , and the values are listed in table 2.

\begin{tabular}{|c|c|c|c|c|}
\hline & $300 \mathrm{~Hz}$ & $350 \mathrm{~Hz}$ & $400 \mathrm{~Hz}$ & Static \\
\hline width of wake $Z / c$ & $3.33 \times 10^{-2}$ & $3.22 \times 10^{-2}$ & $3.45 \times 10^{-2}$ & $3.43 \times 10^{-2}$ \\
\hline
\end{tabular}

Table 2: Wake width for different actuation frequencies, with $Z=\left|z_{1}-z_{2}\right|$. The coordinates $z_{1}$ and $z_{2}$ are chosen along the vertical $z$-axis where $\|\mathbf{u}(z)\|=0.95 U_{\infty}$. 

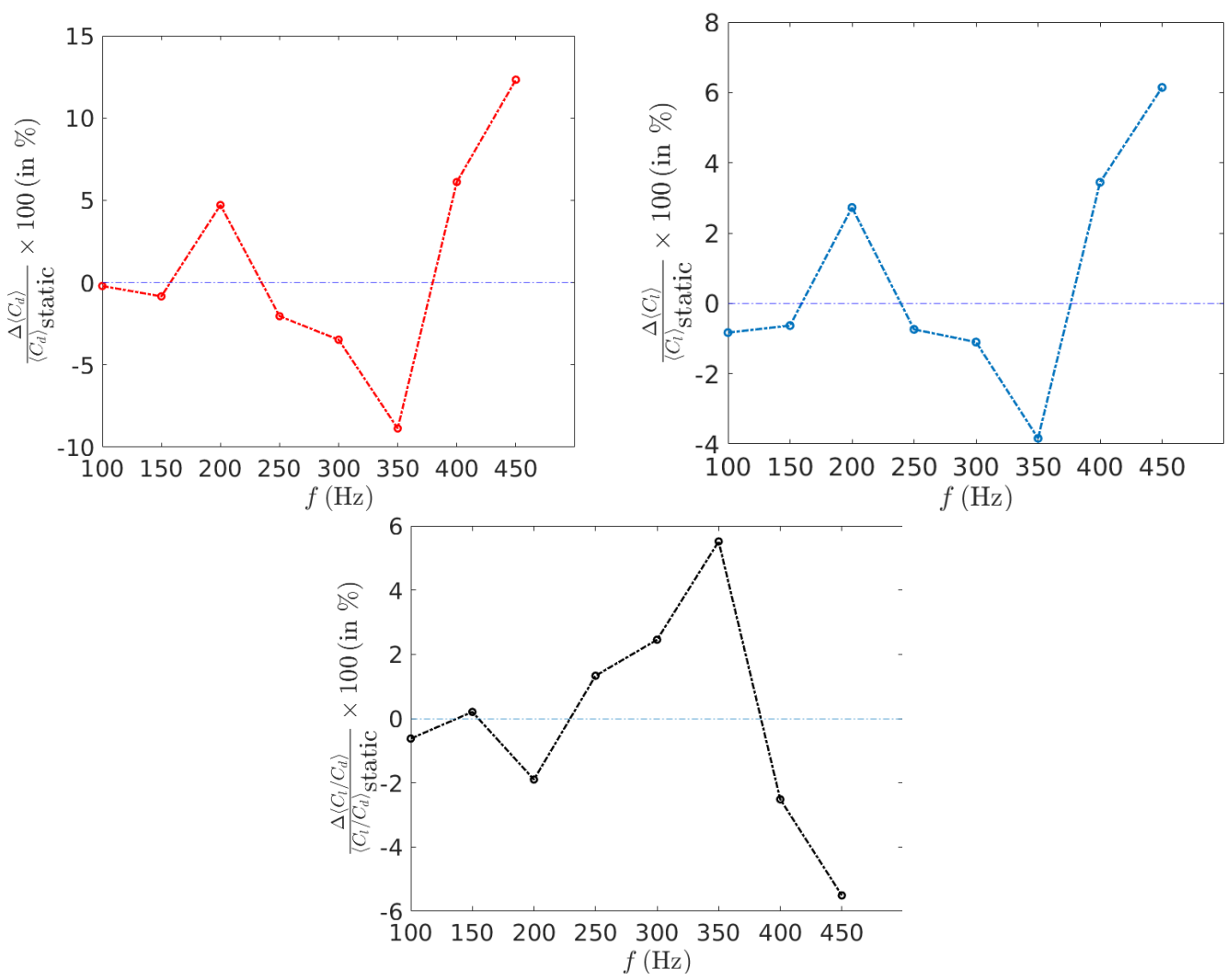

Figure 7: Comparison between time-averaged force coefficients for actuation frequencies $f_{\mathrm{ac}} \leq 450 \mathrm{~Hz}$ and the static case. $100 \times \Delta\left\langle C_{f_{i}}\right\rangle /\left\langle C_{f_{i}}\right\rangle_{\text {static }}=100 \times$ $\left(\left\langle C_{f_{i}}\right\rangle_{\text {morphing }}-\left\langle C_{f_{i}}\right\rangle_{\text {static }}\right) /\left\langle C_{f_{i}}\right\rangle_{\text {static }}$ is the relative mean evolution (in $\%$ ) of the quantity $C_{f_{i}}$. If it is positive, it means that morphing contributes to increase the average value of $C_{f_{i}}$ compared to the static case, and vice versa.

It can be seen from table 3 that the wake becomes thinner in both $f_{\mathrm{ac}}=300 \mathrm{~Hz}$ and $f_{\mathrm{ac}}=350 \mathrm{~Hz}$, and it becomes thicker than the static case wake for an actuation frequency of $400 \mathrm{~Hz}$. This coincides with observations that on average, drag decreases for both $f_{\mathrm{ac}}=300 \mathrm{~Hz}$ and $350 \mathrm{~Hz}$ while it increases for $f_{\mathrm{ac}}=400 \mathrm{~Hz}$. Even more remarkably, the velocity deficit in the case of $f_{\mathrm{ac}}=400 \mathrm{~Hz}$ is more significant than that of the static case, while the velocity deficit for $f_{\mathrm{ac}}=350 \mathrm{~Hz}$ is less important. This indicates that wing morphing achieved by a vibration of the trailing edge acts on the wake dynamics by reducing or increasing total pressure deficit.

\begin{tabular}{|c|c|c|c|}
\hline & $300 \mathrm{~Hz}$ & $350 \mathrm{~Hz}$ & $400 \mathrm{~Hz}$ \\
\hline $100 \times \Delta Z / Z_{\text {static }}$ (in \%) & -2.9 & -6.1 & +0.6 \\
\hline
\end{tabular}

Table 3: Percent change in terms of wake width compared to the static case. $\Delta Z / Z_{\text {static }}=$ $\left(Z_{\text {morphing }}-Z_{\text {static }}\right) / Z_{\text {static }}$ 

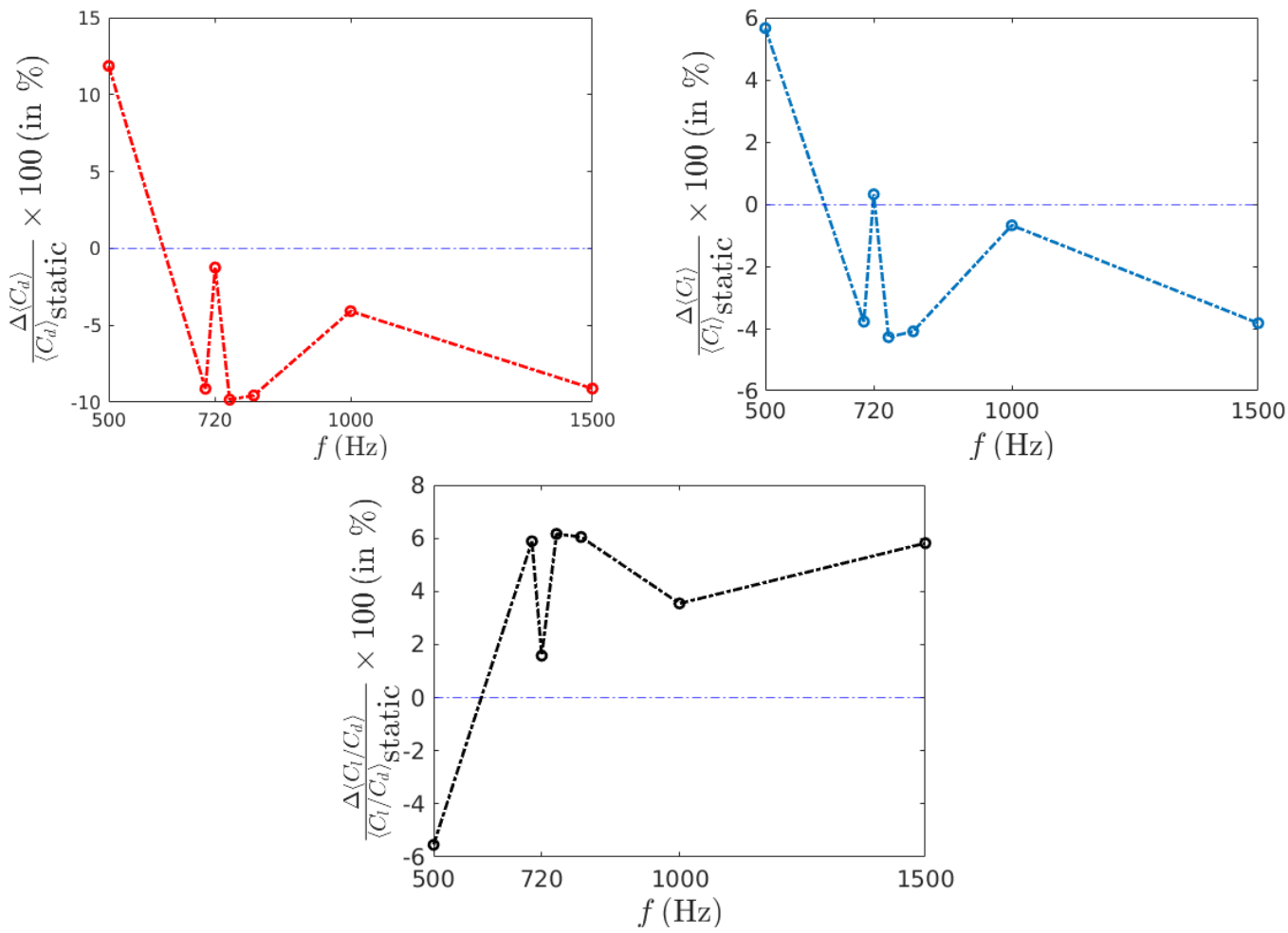

Figure 8: Comparison between time-averaged force coefficients for actuation frequencies $f_{\mathrm{ac}} \geq$ $500 \mathrm{~Hz}$ and the static case.

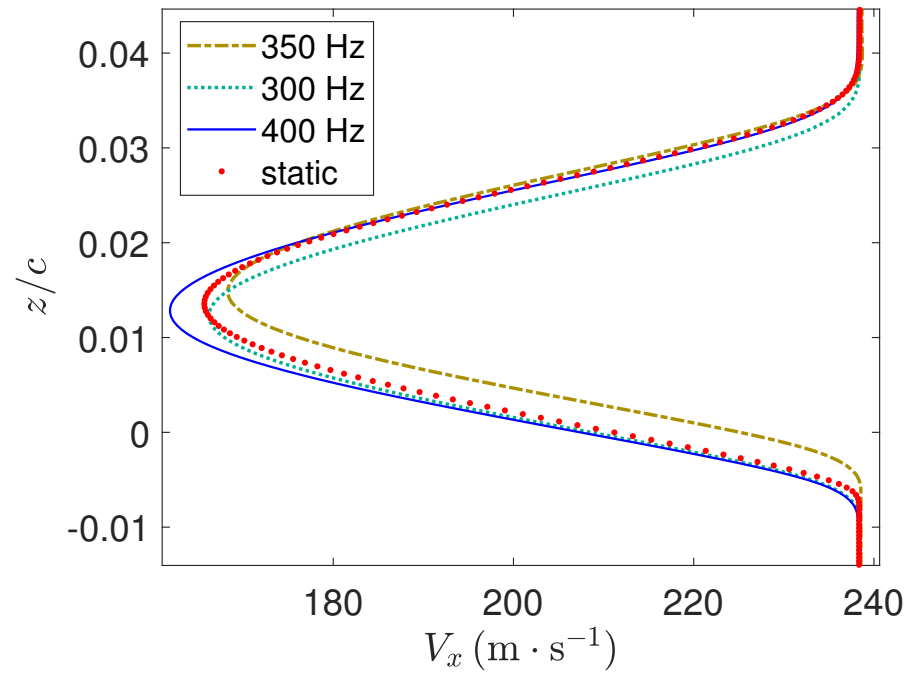

Figure 9: Time-averaged velocity profiles at $x / c=0.19$ for $f_{\text {ac }}=300 \mathrm{~Hz}, 350 \mathrm{~Hz}, 400 \mathrm{~Hz}$ and the static (immobile trailing edge) case.

\section{Conclusions}

This study presents the analysis of trailing edge morphing and its influence on the aerodynamic forces exerted on a supercritical wing in the transonic regime. Due to the presence 
of buffet in this range of Mach numbers which stems from a nascent SWBLI, the investigation also revolves around SWBLI manipulation through hybrid morphing.

Numerical results from 2D computations show that morphing is able to considerably modify the flow dynamics around the airfoil in transonic flight, allowing to suppress force unsteadiness due to buffet with a slight upward deflection of the trailing edge, or to synchronize the instability with a vibration of the wing's aft-end. It has also been shown that wing morphing is capable of changing the pressure distribution in the detached boundary layer and more particularly in the wake region. This contributes to either increase or decrease the width of the wake and the total pressure deficit in this viscous region of the flow, which translates in either a larger or a lower amount of pressure drag characterized by thick separation or a wide turbulent wake.

\section{Acknowledgements}

The authors are grateful to the LAPLACE Laboratory GREM3 research group on electrodynamics and to the National Supercomputing centers CALMIP, CINES and IDRIS for the Computer allocation, as well as the PRACE Supercomputing allocation number 2017174208. This study has been realised under the H2020 European Research programme $\mathrm{n}^{\circ}$ 723402: SMS, "Smart Morphing and Sensing for aeronautical configurations", http: / / smartwing.org/SMS/EU/.

\section{References}

1 Seegmiller, H. L., Marvin, J. G., And Levy, L. L.: Steady and unsteady transonic flow. AIAA Journal, Vol. 16 (1978) 1262-1270.

2 McDevitT, L., LeVy JR., L. And Deiwert, G.: Transonic flow about a thick circular-arc airfoil. AIAA Journal, Vol. 14 (1976) 606-613.

3 LEVY JR., L.: Experimental and computational steady and unsteady transonic flows about a thick airfoil. AIAA Journal, Vol. 16 (1978) 564-572.

4 Marvin, J., LeVy JR., L. And Seegmiller, H.: Turbulence modeling for unsteady transonic flows. AIAA Journal, Vol. 18 (1980) 489-496.

5 Grossi, F., Szubert, D., Braza, M., Sevrain, A., And Hoarau, Y.: Numerical simulation and turbulence modelling of the transonic buffet over a supercritical airfoil at high Reynolds number. Proceedings of the ETMM9 9th International ERCOFTAC Symposium on Engineering Turbulence Modelling and Measurements, Thessaloniki, Greece (2012).

6 JIN, G. AND BRAZA, M.: Two-equation turbulence model for unsteady separated flows around airfoils. AIAA Journal, Vol. 32 (1994) 2316-2320.

7 Braza, M., El Akouri, R., Martinat, G., Hoarau, Y., Harran, G. and Chassaing, P.: Turbulence modelling improvement for highly detached unsteady aerodynamic flows by statistical and hybrid approaches. ECCOMAS CFD 2006: European Conference on Computational Fluid Dynamics, (2006).

8 Szubert D., Grossi F., Jimenez Garcia A., Hoarau Y., Hunt J. C. R. And Braza M.: Shockvortex shear-layer interaction in the transonic flow around a supercritical airfoil at high Reynolds number in buffet conditions. Journal of Fluids and Structures, Vol. 55 (2015) 276-302.

9 Hoarau, Y., Pena, D., Vos, J. B., Charbonier, D., Gehri, A., Braza, M., Deloze, T. And LAUREndeAU, E.: Recent developments of the Navier Stokes Mult Block (NSMB) CFD solver. 54th AIAA Aerospace Sciences Meeting, AIAA SciTech Forum, American Institute of Aeronautics and Astronautics, (2016). 
10 Szubert D., Asproulias, I., Grossi F., Duvigneau, R., HoArau Y. And Braza M.: Numerical study of the turbulent transonic interaction and transition location effect involving optimisation around a supercritical aerofoil. European Journal of Mechanics - B/Fluids, Vol. 55 (2015) 380-393.

11 Tô, J.-B., Simiriotis, N., Marouf, A., Szubert, D., Asproulias, I., Zilli, D., Hoarau, Y., Hunt, J. C. R. AND BRAZA M.: Effects of vibrating and deformed trailing edge of a morphing supercritical airfoil in transonic regime by numerical simulation at high Reynolds number. Journal of Fluids and Structures, (2019).

12 Jodin G., Motta V., Scheller J., Duhayon E., Döll C., Rouchon J.-F. and Braza M.: Dynamics of a hybrid morphing wing with active open loop vibrating trailing edge by Time-Resolved PIV and force measures. Journal of Fluids and Structures, Vol. 74 (2017) 263-290. 